\title{
Scaling of multiplicity distribution in high energy hadron-nucleus interactions
}

\author{
Tufail Ahmad \\ Applied Science \& Humanities Section, University Polytechnic, Faculty of Engineering \& Technology, Aligarh \\ Muslim University, Aligarh-202002, India
}

\begin{abstract}
Analysis of 50 and $340 \mathrm{GeV}$ pion-nucleus and $400 \mathrm{GeV}$ proton- nucleus interactions data for multiplicity scaling study has been carried out. The multiplicity distribution of compound(sum of shower and grey particles in an event), slow (black tracks) particles and target protons (grey tracks) are found to obey a KNO type scaling law.

Keywords: Multiplicity scaling, compound multiplicity, relativistic charged particles, multiparticle production, multiplicity distribution Subject classification PACS 13.85 - Hadron - induced high - and superhigh - energy interactions, energy $>10 \mathrm{GeV}$.
\end{abstract}

\section{Introduction}

During the last many years, the work on high energy experiments was carried out by several workers[1-8] mostly for shower particles which are fast moving charged particles or we can say that relativistic charged particles in hadron-nucleus (hA) and nucleus-nucleus (AA) collisions. The investigation of hA collisions is fundamental for understanding the nature of the interaction process. It is believed that these interactions may provide some very useful information about the dynamics of multiparticle production. An important feature noticed in these interactions is that the nucleus plays the role of target for the incident hadron.

For the present study nuclear emulsion technique has been used to collect the data. Nuclear emulsion is a material which memorises the tracks of charged particles. When a particle, let us call it as a primary particle, is incident on the nuclei of the emulsion, secondary particles are produced in the form of tracks. These particles are termed as shower, grey and black particles; and their number in an event/interaction is given as $n_{s}, n_{g}$ and $n_{b}$.

We have tried to study the compound particle $\left(\mathrm{n}_{\mathrm{c}}\right)$ multiplicity distribution also. A compound particle is defined as the sum of number of shower $\left(n_{s}\right)$ and grey $\left(n_{g}\right)$ particles in an event, i.e., $n_{c}=n_{s}+n_{g}$. The study on compound particle multiplicity was first done by Jurak and Linscheid [9]. We studied some aspects of compound multiplicity distribution in our earlier publication [10], this paper is in continuation to that [10]. Many workers [12-20] have studied various characteristics of compound multiplicity distributions for high energy interactions. An attempt has also been made to study the multiplicity distributions of the slow particles (black tracks) and target protons (grey particles).

In the present paper an effort is made to test the validity of hypothesis of Koba, Neilsen and Olesen [11] which is referred to as KNO scaling in the case of compound as well as slow particles.

\section{Experimental details}

In order to collect the experimental data, a stack of Ilford-G5 emulsion pellicles exposed to a $340 \mathrm{GeV}$ negative pion beam was used. The events/interactions were picked up after leaving $3 \mathrm{~mm}$ from the leading edges of the pellicles to avoid any distortion effects. The measurement was carried out using an oil immersion objective of 100X magnification. To rule out any contamination of primary events with secondary interactions, the primaries of all the events were followed back up to the edge of the plates and only those events whose primary remained parallel to the main direction of the beam and which did not show any significant change in their ionization were finally picked up as genuine primary events.

In these interactions the secondary particles appear in the form of a track. The particles associated with different tracks were classified according to emulsion terminology [21] on the basis of their specific ionization $\mathrm{g}^{*}\left(=\mathrm{g} / \mathrm{g}_{\mathrm{o}}\right)$, where $\mathrm{g}$ is the ionization of the track and $\mathrm{g}_{\mathrm{o}}$ is the ionization of the primary.

The tracks with $\mathrm{g}^{*}<1.4$ are shower tracks. Shower tracks are relativistic charged particles which are mostly produced in the forward cone. The tracks with $1.4 \leq \mathrm{g}^{*} \leq 10$ and $\mathrm{g} *>10$ were named as grey and black tracks respectively. The particles associated with grey and black tracks are also called as slow particles, grey tracks are mostly target protons. In this study two more data sets were used, one at $50 \mathrm{GeV}$ [18] pion-nucleus interactions and the other at $400 \mathrm{GeV}$ [18] proton-nucleus interactions. Thus the analysis is based at three energies i.e., 50, 340 and $400 \mathrm{GeV}$. Other details may be found in our earlier publications[10,18]. 


\section{Results and discussion}

The studies on the multiplicity distribution of charged shower particles produced in hadron-hadron (hh) interactions show that the multiplicity distributions change with energy. However, it is observed that if one plots the distribution in terms of a variable $\mathrm{z}=\mathrm{n}_{\mathrm{s}} /\left\langle\mathrm{n}_{\mathrm{s}}\right\rangle$, then the multiplicity distributions at various energies may be represented by the same mathematical function $\psi(\mathrm{z})$. This fact is observed in hA interactions as well. This means that the multiplicity distribution exhibits a universal behaviour when plotted with $\mathrm{z}$ as the variable. This behaviour of the distribution is known as KNO scaling after Koba, Nielsen and Olesen [11] who first put forward the hypothesis. According to the KNO scaling the probability of observing $\mathrm{n}_{\mathrm{s}}$ charged particles in $\mathrm{pp}$ collisions may be related to a function by

$$
\mathrm{P}_{\mathrm{n}}(\mathrm{s})=\sigma_{\mathrm{n}}(\mathrm{s}) / \sigma_{\text {inel }}(\mathrm{s})=\left(1 /<\mathrm{n}_{\mathrm{s}}>\right) \psi\left(\mathrm{n}_{\mathrm{s}} /<\mathrm{n}_{\mathrm{s}}>\right)=1 /<\mathrm{n}_{\mathrm{s}}>\psi(\mathrm{z})
$$

where $\sigma_{\text {inel }}(\mathrm{s})$ is the total inelastic cross section at a given centre of mass energy, $\sqrt{ }_{\mathrm{s}}$ and $\sigma_{\mathrm{n}}(\mathrm{s})$ is the partial cross section for the production of $\mathrm{n}_{\mathrm{s}}$ charged particles and $\psi(\mathrm{z})$ is an energy independent function at sufficiently high energies. The KNO scaling thus implies that the multiplicity distribution is universal and the function $<\mathrm{n}_{\mathrm{s}}>\mathrm{P}_{\mathrm{n}}(\mathrm{s})$ has no dependence on energy when expressed in terms of a scaling variable $\mathrm{z}$.

Slattery [22] was the first to test the proposed KNO scaling by plotting $<\mathrm{n}_{\mathrm{s}}>\mathrm{P}_{\mathrm{n}}(\mathrm{s})$ as a function of $\mathrm{z}=\mathrm{n}_{\mathrm{s}} /<\mathrm{n}_{\mathrm{s}}>$ for the experimental data on pp collisions in two different ranges of energies, viz., 19 to $28.5 \mathrm{GeV}$ and 50 to $303 \mathrm{GeV}$. Slattery [22] represented the scaling function as follows,

$$
\psi(\mathrm{z})=\left(\mathrm{Az}+\mathrm{Bz}^{3}+\mathrm{Cz}^{5}+\mathrm{Dz}^{7}\right) \exp (-\mathrm{Ez})
$$

The data was fitted by taking the values of the parameters which appears in the functional form as:

$$
\psi(z)=\left(3.79 z+33.7 z^{3}-6.64 z^{5}+0.033 z^{7}\right) \exp (-3.04 z)
$$

The above function was found to give good fit to the pp collision data with $\chi^{2} /$ dof(degree of freedom $)=1.00$ in the energy range 50 to $303 \mathrm{GeV}$ but the fit was poor in the energy range 19 to $28.5 \mathrm{GeV}$ with $\chi^{2} / \mathrm{dof}=2.92$.

Olesen [23] has also observed that KNO scaling law is not well satisfied at very low energies atleast in pp scattering. Buras et al. [24] gave a modified scaling variable,

$$
\mathrm{z}^{\prime}=\left(\mathrm{n}_{\mathrm{s}}-\alpha\right) /\left(<\mathrm{n}_{\mathrm{s}}>-\alpha\right)
$$

which provides an extension of KNO scaling at low energies, i. e., Elab $<50 \mathrm{GeV}$. Here $\alpha$ is an energy independent parameter. The value of $\alpha$ is determined in such a way that the modified KNO scaling function

$$
\psi\left(\mathrm{z}^{\prime}\right)=\left(<\mathrm{n}_{\mathrm{s}}>-\alpha\right)\left(\sigma_{\mathrm{n}}(\mathrm{s}) / \sigma_{\text {inel }}(\mathrm{s})\right)
$$

weakly depends on energy. They [24] gave the following function,

$$
\psi\left(\mathrm{z}^{\prime}\right)=\mathrm{A}\left(\mathrm{z}^{\prime}+\mathrm{B}\right) \exp \left(\mathrm{Cz}^{\prime}+\mathrm{Dz}^{\prime 2}\right)
$$

which they have used to fit the data on pp collisions in the energy range 5.5 to $300 \mathrm{GeV}$ with the values of the parameters as $\mathrm{A}=2.30, \mathrm{~B}=0.142, \mathrm{C}=-0.058$ and $\mathrm{D}=-0.659$. They have taken the value of $\alpha$ as 0.90 .

Martin et al. [25] have tested the KNO scaling in the case of hA interactions taking proton as the projectile. They [25] have shown that the multiplicity distribution of charged particles in proton-nucleus interactions at 30,67 and $300 \mathrm{GeV}$ obeys $\mathrm{KNO}$ scaling law. The functional form given by them is as follows,

$$
\psi(z)=\left(6.84 z+26.6 z^{3}-2.12 z^{5}+0.16 z^{7}\right) \exp (-3.28 z)
$$

the above function was fitted to the data with $\chi^{2} / \mathrm{dof}=0.79$.

Anzon [2] et al. have also used the same function as given by Slattery [22] but with different values of the parameters for pion-nucleus interactions at $200 \mathrm{GeV}$. The values of the fitted parameters in the functional form appear as

$$
\psi(z)=\left(3.0 z+26.0 z^{3}+4.6 z^{5}+0.18 z^{7}\right) \exp (-4.0 z)
$$

El-Nadi et al. [3] have also tested $\mathrm{KNO}$ scaling in pion-nucleus interactions using the function given by Buras et al. [24]. They took the value of $\alpha$ as zero and found the values of the parameters A, B, C and D as $1.15,0.14,0.059$ and -0.659 respectively.

We [7] have also studied KNO scaling for pion-nucleus interactions at 17.2, 50, 200, 300 and $340 \mathrm{GeV}$ in the case of relativistic charged shower particles and tried to fit the function given by Buras et al. We also took the value of $\alpha$ as zero. The values of the parameters obtained by us are $A=1.50 \pm 0.29, B=-0.007 \pm 0.03$, $\mathrm{C}=0.17 \pm 0.13$ and $\mathrm{D}=-0.95 \pm 0.13$ with $\chi^{2} / \mathrm{dof}=0.64$.

Many workers $[13,15,17,20,26-28]$ have tested the KNO scaling law for AA interactions data as well. In the present investigation, we have made an attempt to study $\mathrm{KNO}$ scaling in pion-nucleus interactions at 50 and $340 \mathrm{GeV}$, and proton-nucleus interactions at $400 \mathrm{GeV}$ for compound multiplicity distribution. We have already defined the compound particle in section 1 . In order to test the KNO scaling of multiplicity distribution 
of compound particles, we have calculated the function $\psi(\mathrm{z})$ and the variable $\mathrm{z}$ which has already been defined. We have tried to fit the experimental data at three different energies mentioned above with a scaling function

$$
\psi(\mathrm{z})=\mathrm{Az} \exp (-\mathrm{Bz})
$$

The variation of $\psi(\mathrm{z})$ with $\mathrm{z}$ for compound multiplicity distribution is shown in Figure.1. The solid curve in the figure is due to the above equation with $\mathrm{A}=2.97 \pm 0.12$ and $\mathrm{B}=1.92 \pm 0.32$. The values of the parameters $\mathrm{A}$ and $\mathrm{B}$ were calculated using the CERN standard programme MINUIT and errors as given in MINOS. The value of $\chi^{2} /$ dof is 0.95 . All the experimental points are almost close to the universal curve. Thus we say that the function fits the data quite well. One may conclude here that KNO scaling exists in the given energy range $50-400 \mathrm{GeV}$ for compound particle multiplicity distribution.

An attempt has been made to test the validity of KNO scaling for slow particles i.e. black particles and target protons (grey particles). These are shown in Figures 2 and 3. Same function as appearing in equation (9) was tried to fit the data and the values of the parameters and $\chi^{2} /$ dof obtained are as under $\mathrm{A}=3.37 \pm 0.43, \mathrm{~B}=2.18 \pm 0.13, \chi^{2} / \mathrm{dof}=0.65$ for grey particle multiplicity distribution. $\mathrm{A}=2.52 \pm 0.85, \mathrm{~B}=1.76 \pm 0.27, \chi^{2} / \mathrm{dof}=1.20$ for black particle multiplicity distribution.

It is seen from the figure as well from $\chi^{2}$ /dof that the data is quite well represented by the function with the above values of $\mathrm{A}$ and $\mathrm{B}$. Hence the $\mathrm{KNO}$ scaling is observed in the energy range $50-400 \mathrm{GeV}$ for slow particles and target protons as well. Similar findings have been reported by other workers [26,27] also in AA interactions.

\section{Concluding remarks :}

On the basis of the results discussed in the present investigation, we conclude the following.

The multiplicity distributions of compound particles, slow particles (black tracks) and target protons (grey tracks) are well represented by a single scaling function which indicates the presence of KNO scaling in $\mathrm{hA}$ collisions in the energy range $50-400 \mathrm{GeV}$.

\section{References}

[1]. Z. V. Anzon et al (Alma Ata-Leningrad-Moscow-Tashkent Collaboration), A study of inelastic pion-nucleus interactions at 200 $\mathrm{GeV} / \mathrm{c}$ in an emulsion, Nuclear Physics B, 129, (1977) 205-234.

[2]. I. Otterlund, E. Stenlund, Bo. Andersson and G. Nilson, Nuclear interactions of 400-GeV protons in emulsion, Nuclear Physics B, 142 (1978) 445-462.

[3]. M. El-Nadi, O. E. Badawy, M. K. Hegab et al, Inelastic interactions of $340 \mathrm{GeV} / \mathrm{c} \pi^{-}$with emulsion nuclei, Physical Review D, 27 , (1983) 12-18.

[4]. R. K. Shivpuri and A. Kotha, Multiplicity, rapidity and rapidity correlations in $800 \mathrm{GeV}$ proton-nucleus interactions, Physical Review D, .35, 3508-3511, (1987) 3508-3511.

[5]. A. Tufail, S. Ahmad, A. R. khan, M. Zafar and M. Shafi, Nuclear interactions of 340-GeV pions in emulsion, Physical Review D, 42, (1990) 2187-2193.

[6]. A. Tufail, S. Ahmad and M.Zafar, Features of forward-backward multiplicity correlations in hadronic collisions, Canadian Journal of Physics,74 (1996) 141-143.

[7]. A. Tufail, S. Ahmad, M. Zafar and M. Shafi, Multiplicity correlations and KNO scaling in pion-nucleus interactions, Il Nuovo Cimento A, 104, (1991) 145-150.

[8]. A. Tufail, S. Ahmad, A. R. Khan, M. Zafar and M. Shafi, Particle production in $\pi^{-}$-nucleus collisions at $340 \mathrm{GeV} / \mathrm{c}$, International Journal of modern physics A, 6 (1991) 929-934.

[9]. A. Jurak and A. Linscheid, Some characteristics of compound shower and grey tracks multiplicity distributions produced in proton emulsion interactions, Acta Physica Polonica B, 8 (1977) 875-886.

[10]. T. Ahmad, A study of pion-nucleus interactions in terms of compound particles, ISRN High Energy Physics, 2014 (2014) 1-6.

[11]. Z. Koba, H. B. Nielsen and P. Olesen, Scaling of multiplicity distributions in high energy hadron collisions, Nuclear physics B, 40 (1972) 317-334

[12]. D. Ghosh, J. Roy, K. Sengupta, M. Basu, S. Naha and A. Bhattacharya, A new approach for the study of hadron-nucleus interactions through compound multiplicity, Hadronic Journal, 5 (1981) 163-173.

[13]. T. Ahmad and M. Irfan, Features of compound multiplicity in heavy-ion interactions at $4.5 \mathrm{~A} \mathrm{GeV/c}$, Physical Review C, 44 (1991) $1555-1558$.

[14]. M. S. Khan, S. S. Ali, P. Singh et al., Some interesting results on compound multiplicity in ${ }^{12} \mathrm{C}-$ nucleus reactions at $4.5 \mathrm{~A} \mathrm{GeV} / \mathrm{c}$, Canadian Journal of Physics, 75 (1997) 549-557.

[15]. N. N. Abd Allah and M. Mohery, Features of the compound multiplicity of the interactions of ${ }^{24} \mathrm{Mg}$ and ${ }^{28} \mathrm{Si}$ ions with emulsion nuclei at 4.5 A GeV/c, Turkish Journal of Physics, 25 (2001) 109-119.

[16]. Z. Abou Moussa, Compound multiplicity in the collisions of $4.1 \mathrm{~A} \mathrm{GeV} / \mathrm{c}^{22} \mathrm{Ne}$ nuclei with nuclear emulsion, Canadian Journal of Physics, 80 (2002) 109-117.

[17]. D.-H. Zhang, C.-L. He, Y. Yuan et al., Features of compound multiplicity in ${ }^{16} \mathrm{O}-\mathrm{Em}$ interactions at $4.5 \mathrm{~A}$ GeV/c, Chinese Journal of Physics, 44 (2006) 405-417.

[18]. T. Ahmad, N. Ahmad, S. Ahmad and M. Zafar, Characteristics of compound multiplicity in hadron-nucleus collisions, Indian Journal of pure and Applied Physics, 48 (2010) 855-859.

[19]. M. Mohery and M. Arafa, Some characteristics of the compound multiplicity in high-energy nucleus-nucleus interactions, Internationa Journal of Modern Physics E, 20(2011) 1735-1754.

[20]. C.-Y. Bai and D.-H. Zhang, Study of compound particle production in ${ }^{84} \mathrm{Kr}$-emulsion interactions at $1.7 \mathrm{~A} \mathrm{GeV}$, Chinese Physics C, 35 (2011) 349-354.

[21]. W. H. Barkas, Nuclear Research Emulsions, Academic Press, New York, NY, USA, (1963). 
[22]. P. Slattery, Evidence for the onset of semi-inclusive scaling in proton-proton collisions in the 50-300 GeV/c momentum range, Physical Review Letters, 29 (1972) 1624-1626, ibid, Evidence of the systematic behaviour of charged prong multiplicity distributions in high energy proton-proton collisions, Physical Review D, 7 (1973) 2073-2079.

[23]. P. Olesen, The $200 \mathrm{GeV}$ multiplicity distribution and scaling, Physics Letters B, 41 (1972) 602-604.

[24]. A. J. Buras, J. Dias de Deus and R. Moller, Multiplicity scaling at low energies, a generalized wroblewski-formula and the leading particle effect, Physics Letters B, 47 (1973) 251-254.

[25]. J. W. Martin, J. R. Florian, L. D. Kirkpatrick, J. J. Lord and R. E. Gibbs, Scaling of multiplicity and angular distributions in p emulsion interactions at $30 \mathrm{GeV}, 67 \mathrm{GeV}$ and $200 \mathrm{GeV}$, Il Nuovo Cimento A, 25 (1975) 447-452.

[26]. S. Ahmad, M. A. Ahmad, M. Tariq and M. Zafar, Charged multiplicity distribution in relativistic heavy-ion collisions, International Journal of Modern Physics E, 18 ( 2009) 1929-1944.

[27]. D. Ghosh, A. Deb, S. Bhattacharyya and U. Datta, Multiplicity scaling of target protons in high-energy nucleus-nucleus and hadronnucleus interactions, Journal of Physics G, 39 (2012) 1-11.

[28]. N. S. Chauhan, M. K. Singh, V. Singh and R. Pathak, Characteristics of compound multiplicity in ${ }^{84} \mathrm{Kr}_{36}$ with various light and heavy targets at $1 \mathrm{GeV}$ per nucleon, Indian Journal of Physics, 87 (2013) 1263-1267.

Figure Captions

Fig. 1 Scaling of compound particle multiplicity distribution in pion-nucleus and prton- nucleus interactions.

Fig. 2 Scaling of multiplicity distribution of target protons(grey tracks) in pion-nucleus and protonnucleus interactions.

Fig. 3 Scaling of multiplicity distribution of slow particles(black tracks) in pion-nucleus and protonnucleus interactions.

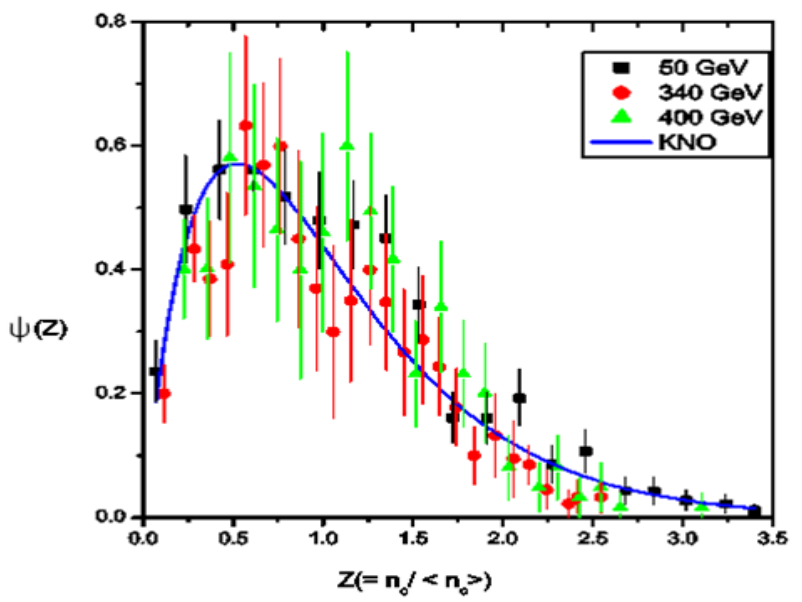

Fig. 1

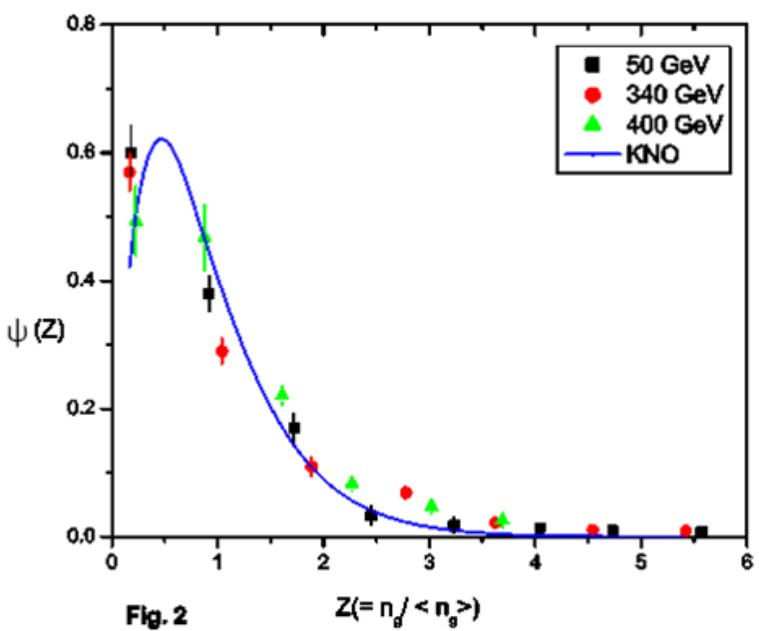




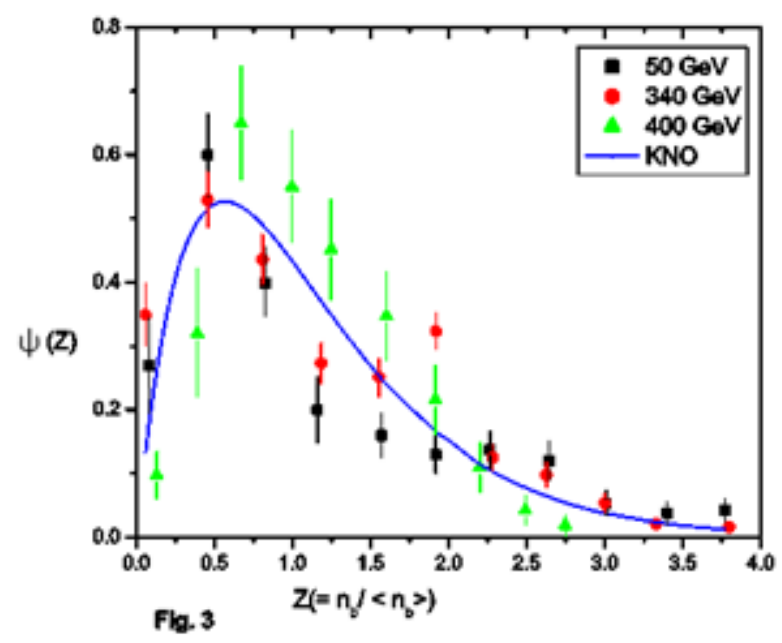

